\title{
Percepción de competencias docentes universitarias desde la perspectiva académica y estudiantil
}

\section{Perception of university teaching skills from the academic and student perspective}

\author{
Rodolfo Mendoza-Llanos \\ Universidad del Bío-Bío, Chile \\ ORCID: http://orcid.org/0000-0001-9529-570X \\ Mauricio Salazar Botello \\ Universidad del Bío-Bío, Chile \\ ORCID: http://orcid.org/0000-0002-5659-7331 \\ Yohana Muñoz Jara \\ Universidad del Bío-Bío, Chile \\ ORCID: http://orcid.org/0000-0003-3897-8840
}

Received 03-24-20 Revised 05-30-20 Accepted 07-07-20 On line 12-04-20

*Correspondence

Email: rmendoza@ubiobio.cl
Cite as:

Mendoza-Llanos, R., Salazar Botello, M., Muñoz Jara, Y. (2020). Percepción de competencias docentes universitarias desde la perspectiva académica y estudiantil. Propósitos y Representaciones, 8(3), e527. doi: http://dx.doi.org/10.20511/pyr2020.v8n3.527 


\section{Resumen}

Un desafío permanente en la educación superior es el desarrollo de competencias docentes en los académicos que permitan responder al nuevo perfil del estudiante, demandando cambios en la forma de impartir y evaluar la docencia. Se evalúa aquí, la percepción de las competencias docentes universitarias desde la perspectiva de los estudiantes y la perspectiva institucional otorgada por los docentes, así como las diferencias por sexo, en una muestra de 1837 estudiantes y 227 académicos de una universidad pública de dos regiones del centro sur de Chile. Los resultados generales indican que docentes dan mayor importancia a las competencias que los estudiantes. Asimismo, mientras los estudiantes valoran las competencias generar un clima agradable en clases e innovar en las prácticas de trabajo, los académicos dan mayor importancia a la transmisión de conocimientos y flexibilizar las evaluaciones. No obstante, existe concordancia entre ambos grupos, al considerar como la competencia menos importante planificar las clases. Respecto las diferencias por sexo, las mujeres (estudiantes y docentes) tienden a dar más importancia a las competencias que los hombres. Los resultados orientan hacia establecer mejores relaciones interpersonales entre estudiantes y docentes más allá de las aulas y por sobre la transmisión de conocimientos en sí misma, considerando diferencias de género.

Palabras clave: Competencias Docentes, Educación Superior, Diferencias de género

\section{Summary}

A permanent challenge in higher education is the development of teaching skills in academics to respond to the new profile of the student, demanding changes in the way teaching is delivered and evaluated. Here we evaluate the perception of university teaching skills from the perspective of students and the institutional perspective provided by teachers, as well as differences by gender, in a sample of 1837 students and 227 academics from a public university in two regions of southcentral Chile. The general results indicate that teachers give greater importance to skills than students. Likewise, while students value skills, generating a pleasant climate in class and innovating in work practices, academics give greater importance to the transmission of knowledge and making evaluations more flexible. Nevertheless, there is agreement between both groups, considering that planning classes is the least important competence. With respect to differences by sex, women (students and teachers) tend to give more importance to skills than men. The results point toward establishing better interpersonal relations between students and teachers beyond the classroom and above the transmission of knowledge itself, considering gender differences.

Keywords: Teaching skills, Higher Education, gender differences

\section{Introducción}

El concepto de competencia, como se conoce actualmente, surge desde los años setentas a partir de los estudios de McClelland (1973), quien lo utilizó para identificar variables que capaces de predecir el rendimiento laboral, estableciendo con ello las primeras comparaciones entre personas que tenían un alto nivel de éxito en su trabajo y aquellas que tenían un rendimiento promedio (o normal). Estos comportamientos son observables en la realidad cotidiana del trabajo y ponen en práctica de manera integrada aptitudes, rasgos de personalidad y conocimientos adquiridos, dejando atrás la importancia que antiguamente se daba a los títulos y diplomas (Fernández, 2007; 
Le Boterf, 2000; Levy-Leboyer, 1995), los cuales además están causalmente relacionados con un estándar de efectividad superior en un trabajo (Spencer \& Spencer, 1993).

El modelo de formación basado en competencias es uno de los temas que ha desafiado la educación superior en los últimos años, y donde la identificación de las competencias que determinan una buena docencia se torna esencial para el mundo de la Educación Superior (López, González, \& De León, 2014; Porto \& Mosteiro, 2014). Pasar de una docencia centrada en contenidos a otra en la cual se fortalecen las competencias generales y las habilidades generales, no sólo del estudiante sino también del docente, debe ser asumida como un compromiso de cambios por ambos. Esta transformación de la docencia debe estar acompañada de una actitud participativa de educadores y educandos, donde se promueva una cultura colaborativa de cambio y se creen programas educativos que permitan movilidad de los estudiantes (González y Wagenaar, 2003).

Estos cambios paradigmáticos, hacen que la concepción del profesor como transmisor y del estudiante como receptor de conocimientos es sustituida por la concepción del docente como orientador, guía que acompaña al estudiante en el proceso de construcción no sólo de conocimientos, sino también en el desarrollo de habilidades y valores asociados a un desempeño profesional eficiente, ético y responsable, y del estudiante como sujeto de aprendizaje (Allard, 1998; Díaz \& Márquez, 2007; R. González \& González, 2007; Gutiérrez \& de Pablos, 2010; Jiménez, Hernández, \& González, 2013; Mertens, 1996; Pedraja, Rodríguez, \& Villarroel, 2012; Riveros, Bohórquez, López, \& Sepúlveda, 2015; Villarroel \& Bruna, 2017).

En este escenario, el docente debe poseer competencias que le permitan manejar una nueva concepción de las capacidades del estudiante, conducente una formación cada vez más integral. Debe ser capaz de generar un progreso real de mejoramiento de aprendizajes significativos, acreditables, relevantes y autónomos (McCauley, Ohlott, \& Ruderman, 1989), otorgando cada vez más protagonismo al estudiante en su proceso de formación (Irigoyen, Jiménez, \& Acuña, 2011; Salas, 2005), transformándose en un apoyo y estimulador del aprendizaje. Este cambio de una educación centrada en el docente a una centrada en el estudiante, redefine los roles de ambos actores y plantea la interrogante de cuáles serían entonces los conocimientos, habilidades y actitudes que debe poseer el docente para el logro de estos propósitos (McCauley, 1989).

Se espera que el docente promueva el desarrollo de competencias como garantía para que los sujetos puedan seguir aprendiendo a lo largo de su vida y se desempeñen de manera pertinente y satisfactoria en un mundo cambiante y complejo. De ahí, la necesidad de definir un nuevo perfil docente que garantice un desempeño profesional eficiente, eficaz y satisfactorio (AlarcónQuinapanta, Freire-Lescano, Pérez-Barral, Frías-Jiménez, \& Nogueira-Rivera, 2019; ÁngelMacías, Ruiz-Díaz, \& Rojas-Soto, 2017; Bozu \& Canto, 2009; Etchegaray, Pascual, \& Calderón, 2018).

Lo anterior plantea un desafío a la educación superior, se requiere pasar de una lógica de la enseñanza que incluye solo la modalidad discursiva a otra en situaciones problema, en donde el docente auspicie, modele, ilustre y retroalimente los desempeños idóneos como alternativas de solución (Irigoyen et al., 2011; Oliveros, 2006). Los docentes se dedican a la enseñanza suelen inclinarse a favor de la construcción teórica, la generalización y la ideación creativa por encima de la transmisión de habilidades prácticas (Bok, 1992; Bozu \& Canto, 2009; Fernandes, Sotolongo, \& Martínez, 2016; Posada, 2004). 
Para Segura (2004) esta nueva visión de la universidad supone la presencia de ciertos rasgos fundamentales en el perfil integral del docente, entre los cuales destacan, la capacidad de adaptación permanente a las nuevas circunstancias y demandas del entorno social. Las nuevas exigencias obligan a transformar a los estudiantes, lo que significa ayudar al estudiante a descubrir y convertirse en un ser nuevo, capaz de comprender y liderar el cambio (Clemente-Ricolfe \& Escribá-Pérez, 2013; Quinn, Faerman, Thompson, \& McGrath, 1994). Se trata de que las universidades sean capaces de lograr la comprensión, que es al mismo tiempo, medio y fin de la comunicación humana, porque reformar las mentalidades es la tarea de la educación (Blašková, Blaško, \& Kucharčíková, 2014; Corvalán, 2009; Jiménez et al., 2013). Para lograr este cambio, las instituciones educativas deben asumir una responsabilidad académica diferente a la tradicional y construir un proyecto novedoso que incluya el desarrollo teórico, la integración de las funciones sustantivas, los planteamientos curriculares, el énfasis en la formación docente y los aspectos didácticos, incorporando las actuales demandas laborales sin descuidar la formación integral de los estudiantes (Chadwick, 1983; Fugellie, 2009; Fugellie, Rodríguez, \& Yupanqui, 2009; Huerta, Pérez, \& Castellanos, n.d.; Martínez, 2008; Salazar \& Chiang, 2007; Salazar \& Yobánolo, 2002; Yáñez).

Hoy se busca replantear la docencia no sólo en cuestiones académicas y disciplinarias, sino mediante el desarrollo del enfoque de las competencias docentes para retomar la docencia como una actividad profesional (Vargas, 2017; Zabalza, 2003). La mayoría de los profesores universitarios carecen de estudios formales de pedagogía y se diferencian del resto de niveles de enseñanza, en dos aspectos esenciales, a la vez que imparten clases e investigan, y esta función investigativa no solo está relacionada con el contenido de las asignaturas y disciplinas que imparte, sino que también sobre su labor educativa (Ibáñez-Martín, 1990; Rumbo, 1998; Tigelaar, Dolmans, Wolfhagen, \& Van Der Vleuten, 2004).

Según Asún, Rapún, \& Romero (2019) el aprendizaje basado en competencias constituye hoy en día el núcleo central de la docencia en Educación Superior. Sin embargo, todavía se siguen desarrollando más las competencias que definen el perfil profesional que las genéricas. Aspecto sobre el cual se debe reflexionar, para acercar las posturas intereses de los estudiantes y docentes.

Aunque todo lo anterior tiene una clara - y bien intencionada - orientación pedagógica e instrumental en el desarrollo de las futuras carreras profesionales de los estudiantes, no se debe soslayar que los intereses, necesidades y aspiraciones de los estudiantes y profesores no necesariamente son los mismos, por lo tanto no podemos esperar que la visión de las competencias requeridas también lo sean (Gutiérrez-Diez, Piñón, \& Sapién, 2020; Moreno-Murcia \& Silveira, 2015), sobre todo si hoy, estamos frente a un "nuevo alumnado" que accede a la universidad arrastrando carencias sociales y económicas (Arza, De Salvador \& Mascarenhas, 2014) que incluso pueden agudizarse en ella (Salazar-Botello, Mendoza-Llanos \& Muñoz-Jara, 2020).

Desde este punto de vista y al igual que plantea Gómez (2016), el presente estudio pretende recoger la percepción de las competencias docentes universitarias desde la perspectiva de los estudiantes - en la línea de estudios subalternos-, para compensar los debates que se puedan generar respecto de estudios de competencias docentes basados en la perspectiva institucional (Porto \& Mosteiro, 2014). En este sentido consideramos importante diferenciar estas evaluaciones por sexo, considerando la evidencia existente respecto de dificultades en el desarrollo de carreras para las mujeres, tanto académicas (Baeza Reyes \& Lamadrid Álvarez, 2019) como para estudiantes (Arza, De Salvador \& Mascarenhas, 2014) las que recogidas en diferencias estamentales pueden quedar invisibilizadas. 


\section{Método}

La investigación es cuantitativa, no experimental y de corte transversal. La muestra de esta investigación corresponde a estudiantes universitarios $(n=1837)$ y académicos $(n=227)$ de una universidad pública de dos regiones centro sur de Chile. Los participantes se seleccionaron a través de un muestreo no probabilístico por conveniencia, donde el criterio de selección, en el caso de los académicos, estaba dado por ser académico(a) jornada completa o media jornada perteneciente a una de las 6 facultades de la Universidad. En el caso de los estudiantes, estos pertenecían a distintos niveles de formación a 32 carreras de pregrado de seis facultades distintas de la universidad.

Tabla 1. Distribución y estadísticos descriptivos de años en la institución de la muestra en la institución en años según facultad de pertenencia

\begin{tabular}{|c|c|c|c|c|c|c|c|c|c|c|c|}
\hline \multirow{3}{*}{ Facultad } & \multicolumn{5}{|c|}{ Estudiantes } & \multicolumn{6}{|c|}{ Académicos } \\
\hline & \multicolumn{3}{|c|}{ Mujeres } & \multicolumn{2}{|c|}{ Hombres } & \multicolumn{3}{|c|}{ Mujeres } & \multicolumn{3}{|c|}{ Hombres } \\
\hline & $f$ & $M$ & $D E$ & $f$ & $M \quad D E$ & $f$ & $M$ & $D E$ & $f$ & $M$ & $D E$ \\
\hline $\begin{array}{l}\text { Educación y } \\
\text { Humanidades }\end{array}$ & 377 & 2,19 & 1,43 & 204 & $2,311,39$ & 30 & 10,74 & 10,83 & 34 & 16,46 & 12,55 \\
\hline Ciencias Empresariales & 135 & 2,53 & 1,86 & 234 & $2,901,86$ & 10 & 13,70 & 3,86 & 26 & 14,35 & 7,07 \\
\hline $\begin{array}{l}\text { Cs. de la Salud y de los } \\
\text { Alimentos }\end{array}$ & 142 & 2,48 & 1,56 & 33 & $2,691,64$ & 10 & 19,80 & 13,28 & 5 & 12,92 & 11,00 \\
\hline $\begin{array}{l}\text { Arquitectura, } \\
\text { Construcción y Diseño }\end{array}$ & 103 & 2,58 & 1,81 & 150 & $2,511,77$ & 8 & 11,44 & 6,73 & 28 & 16,96 & 9,38 \\
\hline Ciencias & 26 & 1,81 & 1,44 & 24 & $1,711,12$ & 5 & 33,60 & 6,88 & 28 & 18,18 & 12,33 \\
\hline Ingeniería & 48 & 3,28 & 2,39 & 361 & $2,371,90$ & 6 & 19,25 & 11,29 & 37 & 19,93 & 11,13 \\
\hline Total & 831 & & & 1006 & & 69 & & & 158 & & \\
\hline
\end{tabular}

\section{Instrumentos}

Se utilizó la Escala de Competencias Docentes de Salazar, Chiang, \& Muñoz (2016) que cuenta con 50 ítems en formato tipo likert donde 1 = "Sin Importancia" y 5 = "Muy Importante". Contiene dos factores y trece subdimensiones: Innovar en el proceso de enseñanzaaprendizaje (Innovar en las prácticas de trabajo; Construcción de relaciones; Comprensión Interpersonal; Perspicacia Interpersonal; Compromiso Institucional y, Búsqueda de información y profundización del conocimiento) e, Impartir Docencia (Establecimiento de Normas; 
Responsabilidad; Transmisión de Conocimientos; Flexibilizar las evaluaciones; Entregar Retroalimentación; Generar un clima agradable de clases; y Planificar las Clases).

Además, se consideran preguntas sociodemográficas: sexo y años de pertenencia en la institución.

\section{Procedimiento}

El instrumento se aplicó en su versión completa. La recolección de los datos se realizó de manera presencial a todos los participantes. En el caso de los académicos, la recolección de los datos se realizó mediante visitas en sus oficinas. En el caso de los estudiantes, el cuestionario fue aplicado de manera colectiva en las salas de clases de todas las carreras vigentes de la universidad, previa autorización de los directores de escuela ${ }^{1}$, previa firma del consentimiento informado.

\section{Análisis de datos}

Para evaluar confiabilidad se calcularon: Omega de McDonald, Alfa de Cronbach, sus intervalos de confianza y correlación interitem de acuerdo Domínguez (2012).

Para responder a los objetivos del estudio se realizan comparación de medias mediante la prueba $t$ de Student y su respectivo cálculo de tamaño del efecto mediante d de Cohen, los cuales se interpretaron según la propuesta de Erceg-Hurn y Mirosevich (2008) donde efectos pequeño, mediano y grande, se interpretan con indicadores de, $20 ;, 50$ y ,80 respectivamente.

Todos los análisis se realizaron mediante el software JASP 0.11.1 (JASP Team, 2019).

\section{Resultados}

Respecto de la confiabilidad del instrumento (Tabla 1), en su globalidad presenta una confiabilidad aceptable $(\alpha=, 95)$ dada la variabilidad de competencias que se están consultando. Esta confiabilidad da cuenta del constructo de Competencias Docentes en sus dos factores coherentes, al cubrir de mejor forma el contenido del constructo que se entiende multidimensional (Oyanedel, Vargas, Mella \& Páez, 2017).

${ }^{1}$ El director de escuela es quien administra el currículo de una carrera en particular en la universidad. 
Tabla 2. Confiabilidad de las dimensiones de las Competencias Docentes

\begin{tabular}{|c|c|c|c|c|c|c|}
\hline \multirow{3}{*}{$\begin{array}{l}\text { Innovar en el proceso de enseñanza-aprendizaje } \\
\end{array}$} & \multirow{3}{*}{$\begin{array}{c}\mathrm{N}^{\mathrm{o}} \mathrm{de} \\
\text { ítems }\end{array}$} & \multirow{3}{*}{$\begin{array}{c}\omega \\
, 90\end{array}$} & \multirow{3}{*}{$\begin{array}{c}\alpha \\
, 90\end{array}$} & \multicolumn{2}{|c|}{ IC $95 \% \alpha$} & \multirow{2}{*}{$\begin{array}{l}\text { Correlación } \\
\text { interitem }\end{array}$} \\
\hline & & & & $\begin{array}{c}\text { Inferio } \\
\mathrm{r}\end{array}$ & & \\
\hline & & & & ,89 & ,91 & ,29 \\
\hline Innovar en Prácticas de Trabajo & 4 & ,64 & ,64 & ,61 & ,66 & ,31 \\
\hline Construcción de Relaciones & 3 & ,72 &, 72 & ,69 & ,74 & ,46 \\
\hline Comprensión Interpersonal & 3 & ,64 &, 59 &, 54 & ,60 &, 32 \\
\hline Perspicacia Interpersonal & 3 & ,73 & ,72 & ,69 & ,73 & ,46 \\
\hline Compromiso Institucional & 4 &, 76 & ,76 & ,74 & ,77 &, 44 \\
\hline $\begin{array}{l}\text { Búsqueda de Información y profundización del } \\
\text { Conocimiento }\end{array}$ & 6 &, 81 &, 81 & ,79 &, 82 &, 41 \\
\hline Impartir Docencia & 27 & ,92 & ,92 & ,91 & ,92 & ,30 \\
\hline Establecimiento de Normas & 4 &, 80 &, 80 & ,78 &, 81 & ,49 \\
\hline Responsabilidad & 3 & ,73 &, 73 &, 71 &, 75 &, 47 \\
\hline Transmisión de Conocimientos & 3 & ,68 & ,66 & ,64 & ,69 &, 40 \\
\hline Flexibilizar las Evaluaciones & 5 & ,66 & ,66 & ,60 & ,65 &, 28 \\
\hline Entregar Retroalimentación & 3 & ,68 & ,66 & ,63 & ,68 & ,39 \\
\hline Generar Clima Agradable de clases & 3 &, 73 &, 72 &, 70 &, 74 & ,46 \\
\hline Planificar las clases & 6 &, 81 &, 81 &, 79 & ,82 & ,41 \\
\hline
\end{tabular}

$\mathrm{n}=2067$.

La comparación de medias de las evaluaciones en la percepción de competencias entre estudiantes y académicos (Tabla 3 ) da cuenta de que existen diferencias significativas en todas las competencias evaluadas, siendo las puntuaciones mayores en los académicos que en los estudiantes, excepto en la competencia Planificar Clases. Estas diferencias son de un efecto mediano en Responsabilidad, y en las otras, un tamaño de efecto pequeño. 
Tabla 3. Comparación de medias entre estudiantes y académicos

\begin{tabular}{|c|c|c|c|c|c|c|c|}
\hline & \multicolumn{2}{|c|}{ Estudiantes } & \multicolumn{2}{|c|}{ Académicos } & \multirow[b]{2}{*}{$t$} & \multirow[b]{2}{*}{$p$} & \multirow[b]{2}{*}{$D$} \\
\hline & $M$ & $D E$ & $M$ & $D E$ & & & \\
\hline Innovar en Prácticas de Trabajo & 4,39 & 0,54 & 4,50 & 0,50 & $-2,81$ &, 01 & $-0,20$ \\
\hline Construcción de Relaciones* & 3,75 & 0,79 & 3,89 & 0,69 & $-2,67$ &, 01 & $-0,19$ \\
\hline Comprensión Interpersonal* & 4,05 & 0,71 & 4,40 & 0,57 & $-7,12$ & $<, 001$ & $-0,50$ \\
\hline Perspicacia Interpersonal* & 4,31 & 0,61 & 4,56 & 0,46 & $-5,84$ & $<, 001$ & $-0,41$ \\
\hline Compromiso Institucional & 4,00 & 0,66 & 4,16 & 0,63 & $-3,35$ & $<, 001$ & $-0,24$ \\
\hline $\begin{array}{l}\text { Búsqueda de Información y profundización del } \\
\text { Conocimiento* }\end{array}$ & 4,20 & 0,60 & 4,45 & 0,48 & $-6,14$ & $<, 001$ & $-0,43$ \\
\hline Establecimiento de Normas* & 4,37 & 0,60 & 4,65 & 0,48 & $-6,97$ & $<, 001$ & $-0,49$ \\
\hline Responsabilidad* & 4,34 & 0,67 & 4,69 & 0,43 & $-7,63$ & $<, 001$ & $-0,54$ \\
\hline Transmisión de Conocimientos* & 4,28 & 0,62 & 4,49 & 0,47 & $-4,95$ & $<, 001$ & $-0,35$ \\
\hline Flexibilizar las Evaluaciones & 4,28 & 0,56 & 4,36 & 0,52 & $-2,10$ &, 04 & $-0,15$ \\
\hline Entregar Retroalimentación* & 4,28 & 0,63 & 4,49 & 0,51 & $-4,70$ & $<, 001$ & $-0,33$ \\
\hline Generar Clima Agradable de clases & 4,41 & 0,58 & 4,52 & 0,54 & $-2,85$ &, 00 & $-0,20$ \\
\hline Planificar las clases & 4,32 & 0,58 & 4,35 & 0,50 & $-0,73$ & ,47 & $-0,05$ \\
\hline
\end{tabular}

*No se han asumido varianzas iguales $(\mathrm{gl}=2065)$

Al comparar por sexo entre académicos (Tabla 4), no se observan diferencias significativas en Construcción de Relaciones. Respecto de la dimensión Impartir Docencia, no existen diferencias en varias competencias, que jerarquizadas de acuerdo a las medias alcanzadas, de mayor a menor importancia son: Establecimiento de Normas, Responsabilidad, Generar clima agradable de clases, Entregar retroalimentación, Transmisión de conocimiento y Planificar clases. Las diferencias entre académicos mujeres y hombres, se concentran en la dimensión Innovar en el proceso de enseñanza-aprendizaje, siendo las mujeres las que presentan los puntajes más altos de ambos grupos. En orden de mayor a menor importancia se encuentran Perspicacia Interpersonal, Innovar en prácticas de trabajo, Búsqueda de Información y profundización del Conocimiento, Comprensión Interpersonal y Compromiso Institucional. 
Tabla 4. Comparación de medias entre académicos mujeres y hombres

\begin{tabular}{|c|c|c|c|c|c|c|c|}
\hline & \multicolumn{2}{|c|}{ Mujeres } & \multicolumn{2}{|c|}{ Hombres } & \multirow[b]{2}{*}{$t$} & \multirow[b]{2}{*}{$p$} & \multirow[b]{2}{*}{$d$} \\
\hline & $M$ & $D E$ & $M$ & $D E$ & & & \\
\hline Innovar en Prácticas de Trabajo* & 4,63 & 0,37 & 4,44 & 0,53 & 2,60 &, 01 & 0,37 \\
\hline Construcción de Relaciones & 3,96 & 0,65 & 3,86 & 0,71 & 1,01 &, 31 & 0,15 \\
\hline Comprensión Interpersonal & 4,51 & 0,55 & 4,35 & 0,57 & 1,94 &, 05 & 0,28 \\
\hline Perspicacia Interpersonal* & 4,67 & 0,40 & 4,51 & 0,48 & 2,38 &, 02 & 0,34 \\
\hline Compromiso Institucional & 4,29 & 0,54 & 4,10 & 0,66 & 2,10 &, 04 & 0,30 \\
\hline $\begin{array}{l}\text { Búsqueda de Información y profundización del } \\
\text { Conocimiento* }\end{array}$ & 4,60 & 0,37 & 4,39 & 0,50 & 3,04 &, 00 & 0,44 \\
\hline Establecimiento de Normas & 4,72 & 0,40 & 4,63 & 0,51 & 1,38 &, 17 & 0,20 \\
\hline Responsabilidad & 4,73 & 0,37 & 4,67 & 0,46 & 0,83 &, 41 & 0,12 \\
\hline Transmisión de Conocimientos & 4,51 & 0,44 & 4,48 & 0,48 & 0,47 & ,64 & 0,07 \\
\hline Flexibilizar las Evaluaciones* & 4,47 & 0,40 & 4,31 & 0,56 & 2,16 &, 03 & 0,31 \\
\hline Entregar Retroalimentación & 4,57 & 0,46 & 4,46 & 0,53 & 1,53 &, 13 & 0,22 \\
\hline Generar Clima Agradable de clases & 4,58 & 0,46 & 4,49 & 0,57 & 1,16 &, 25 & 0,17 \\
\hline Planificar las clases & 4,44 & 0,47 & 4,30 & 0,52 & 1,86 &, 06 & 0,27 \\
\hline
\end{tabular}

*No se han asumido varianzas iguales $(\mathrm{gl}=225)$ Mujeres $=69 ;$ Hombres $=158$

Al comparar por sexo entre estudiantes (Tabla 5), las diferencias son estadísticamente significativas en todas las competencias, siendo los puntajes mayores en el grupo de estudiantes mujeres que en el de los hombres. En orden de prioridad basado en los puntajes, respecto del proceso de enseñanza-aprendizaje, los estudiantes dan importancia Innovar en prácticas de trabajo, perspicacia interpersonal, búsqueda de información y profundización del conocimiento, comprensión interpersonal, compromiso institucional y construcción de relaciones. Respecto de la forma de impartir docencia, las prioridades son generar un clima agradable de clases, el establecimiento de normas, la responsabilidad, la planificación de las clases, la entrega de retroalimentación, flexibilizar las evaluaciones y transmisión de conocimientos. 
Tabla 5. Comparación de medias entre estudiantes mujeres y hombres

\begin{tabular}{|c|c|c|c|c|c|c|c|}
\hline & \multicolumn{2}{|c|}{ Mujeres } & \multicolumn{2}{|c|}{ Hombres } & \multirow[b]{2}{*}{$t$} & \multirow[b]{2}{*}{$p$} & \multirow[b]{2}{*}{$d$} \\
\hline & $M$ & $D E$ & $M$ & $D E$ & & & \\
\hline Innovar en Prácticas de Trabajo* & 4,48 & 0,47 & 4,32 & 0,58 & 6,08 & $<, 001$ & 0,29 \\
\hline Construcción de Relaciones* & 3,87 & 0,73 & 3,64 & 0,82 & 6,35 & $<, 001$ & 0,30 \\
\hline Comprensión Interpersonal* & 4,18 & 0,66 & 3,93 & 0,74 & 7,50 & $<, 001$ & 0,35 \\
\hline Perspicacia Interpersonal* & 4,39 & 0,56 & 4,25 & 0,64 & 5,21 & $<, 001$ & 0,24 \\
\hline Compromiso Institucional & 4,07 & 0,64 & 3,95 & 0,67 & 3,95 & $<, 001$ & 0,19 \\
\hline $\begin{array}{l}\text { Búsqueda de Información y profundización del } \\
\text { Conocimiento* }\end{array}$ & 4,31 & 0,55 & 4,11 & 0,62 & 7,03 & $<, 001$ & 0,33 \\
\hline Establecimiento de Normas* & 4,48 & 0,55 & 4,27 & 0,62 & 7,52 & $<, 001$ & 0,35 \\
\hline Responsabilidad* & 4,45 & 0,60 & 4,25 & 0,71 & 6,15 & $<, 001$ & 0,29 \\
\hline Transmisión de Conocimientos* & 4,37 & 0,56 & 4,20 & 0,65 & 6,10 & $<, 001$ & 0,29 \\
\hline Flexibilizar las Evaluaciones* & 4,39 & 0,50 & 4,18 & 0,58 & 8,17 & $<, 001$ & 0,38 \\
\hline Entregar Retroalimentación* & 4,40 & 0,57 & 4,19 & 0,67 & 6,96 & $<, 001$ & 0,33 \\
\hline Generar Clima Agradable de clases* & 4,51 & 0,50 & 4,32 & 0,62 & 7,11 & $<, 001$ & 0,33 \\
\hline Planificar las clases* & 4,41 & 0,53 & 4,23 & 0,61 & 6,71 & $<, 001$ & 0,31 \\
\hline
\end{tabular}

$*$ No se han asumido varianzas iguales $(\mathrm{gl}=1835)$ Mujeres $=831 ;$ Hombres $=1006$

Los resultados de las comparaciones se pueden observar en la Fig. 1, en ella, las tendencias por sexo dan cuenta que, aunque el grupo de académicos tiene puntajes mayores al del grupo de estudiantes, las estudiantes mujeres tienen puntajes similares a los académicos en las competencias relacionadas con la necesidad de generar un clima agradable de clases, planificarlas y flexibilizar las evaluaciones. Los estudiantes hombres presentan los puntajes más bajos en todas las competencias. 


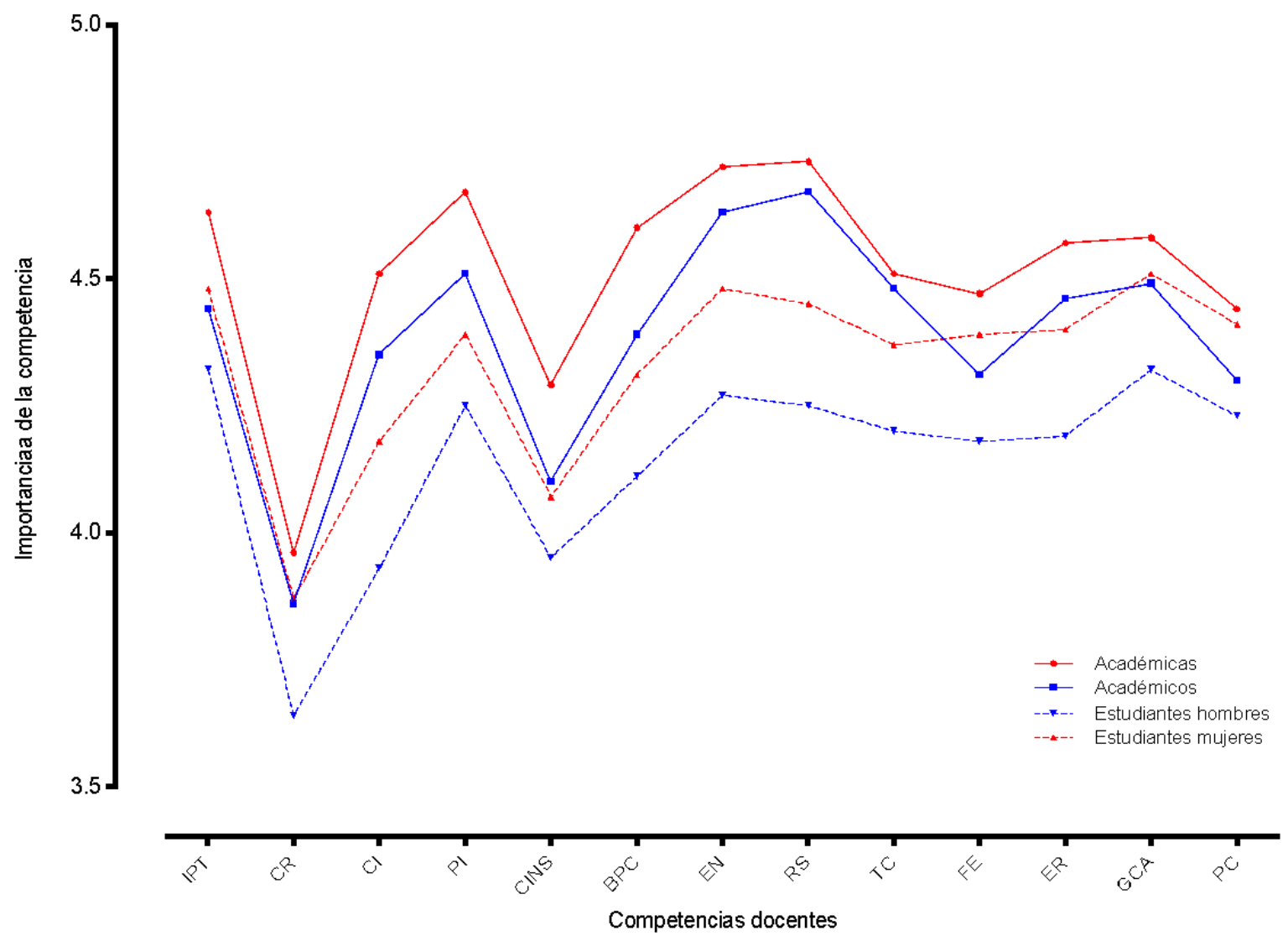

\section{Discusión}

El desarrollo de las competencias docentes es un desafío que las universidades han enfrentado con el desarrollo de planes y programas sistemáticos que buscan mejorar el proceso de enseñanzaaprendizaje de sus estudiantes (Hurtado Espinoza \& Madueño Serrano, 2017; Porto \& Mosteiro, 2014) con miras a responder a las exigencias de las nuevas generaciones de alumnos, pero usualmente desde una perspectiva institucional. Desde esta perspectiva, nuestros resultados son los esperados, puesto que se encontraron diferencias entre docentes y estudiantes, lo que concuerda con la literatura (Gutiérrez-Diez, Piñón, \& Sapién, 2020; Moreno-Murcia \& Silveira, 2015), y donde los docentes dan mayor importancia a todas las competencias que los estudiantes. Esto podría entenderse desde la perspectiva del rol con la que los académicos asumen la docencia y asociarse también con un sesgo de autoservicio (Sachau, 2007) por parte de ellos.

Sin embargo, la concordancia entre la visión de estudiantes y académicos, en la competencia "planificar las clases", parece dar cuenta de que no es prioridad ni para estudiantes ni para docentes, lo que coincide con un estudio previo en el mismo contexto (Salazar et al., 2016). Si bien la planificación de clases es una exigencia explícita para el cumplimiento administrativo y logro de cumplimiento de los programas de asignatura, probablemente estas planificaciones deberían contemplar la incorporación de los atributos relacionales (Gómez, 2016) discutidos anteriormente, como actividad transversal.

Aun cuando existen diferencias entre estudiantes y docentes, ambos grupos establecen con los puntajes más altos las mismas competencias docentes, destacando entre ellas a la "Innovar 
en prácticas de trabajo", "Establecimiento de normas" y "Responsabilidad". Es interesante ver la coincidencias en las valorizaciones, lo cual permite reflexionar respecto cuán competentes deben ser efectivamente los docentes o que esperan los estudiantes de la docencia, teniendo en consideración las nuevas generaciones, realidades y exigencias (Gómez, 2016).

Respecto las competencias relacionadas con "Impartir Docencia", se evidencia se requiere de una formación mucho más allá de la sola formación profesional disciplinaria, considerando que la mayoría de los profesionales que se dedica a la docencia universitaria no tienen formación pedagógica. Lo que se considera menos relevante en este sentido para ambos grupos es la "Planificación de las Clases", sin embargo los temas, tales como la responsabilidad, la entrega de retroalimentación, el establecimiento de normas y la transmisión de conocimientos se destacan como importantes. Existe un profundo conocimiento de las implicancias de esta labor, donde más allá de las exigencias propias de la academia (investigación, transferencia tecnológica, extensión, entre otras), se vislumbra la docencia es lo fundamental, pero que no puede estar desvinculada de las otras funciones (López, González \& De León, 2014).

En las comparaciones por sexo, las académicas dan más importancia a las competencias que los académicos, teniendo una relación significativa en 6 de ellas. Innovar en las prácticas de trabajo, comprensión interpersonal, perspicacia intrapersonal, compromiso institucional, búsqueda de información y profundización del conocimiento y flexibilizar las evaluaciones. Llama la atención que 4 de estas competencias están relacionadas con "Innovar en el procesos enseñanza aprendizaje", esto puede indicar una mayor preocupación por mejorar los procesos y servicios implícitos de la enseñanza aprendizaje, estar abiertas a la retroalimentación, escuchar activamente, manteniendo una preocupación activa por las necesidades de los estudiantes, reconoce la importancia de la formación y de su rol como docente, demostrando involucramiento con las actividades académicas y la institución.

Al revisar la comparación de las medias entre estudiantes mujeres y hombres, las mujeres presentan promedios mayores que los hombres en todas las competencias evaluadas. Específicamente, los promedios más altos están relacionados con aquellas competencias relacionadas con "Impartir Docencia". Esto muestra que para ellas es importante el establecimiento de normas, que se entreguen claramente las condiciones en las cuales se realizar una asignatura. La responsabilidad que demuestra el docente, tras cumplir con las normas que él mismo define para su curso. Valoran en el docente la capacidad para entregar el conocimiento, con claridad, demostrando habilidades comunicacionales. Esperan sea capaz de flexibilizar las evaluaciones, que construyan evaluaciones que se transformen en una instancia de aprendizaje y que permitan efectivamente verificar si se cumplen los resultados de aprendizaje previstos. Abierto a entregar retroalimentación a partir de la objetividad y de una escucha activa. Crea espacios de trabajo en clases que permiten la comunicación, participación y confianza y que sea capaz de planificar de manera ordenada las actividades relacionadas con la asignatura, definiendo calendarios, objetivos, metodología y evaluaciones. Los promedios son semejantes en ambos grupos, se puede apreciar el énfasis en las mismas competencias, pero tras esta comparación se destaca el promedio de las estudiantes mujeres.

Las comparaciones por sexo dan cuenta de una relación interesante que es importante visibilizar. Mientras que en los docentes, en general las mujeres valoran más las competencias asociadas a "Innovar en el proceso de enseñanza-aprendizaje" que los hombres docentes, las mujeres estudiantes valoran más todas las competencias que los estudiantes hombres. Desde una perspectiva de género, estas diferencias en la valoración de competencias docentes son siempre 
mayores en las mujeres, las que podrían estar relacionadas con la presión por relaciones sociales asociadas a la exigencia en el desempeño (Baeza Reyes \& Lamadrid Álvarez, 2019) y por tanto, derribar "barreras internas" en la universidad que reproducen las existentes en la sociedad. Sobre este mismo punto, el desequilibrio que se observa según distribución por sexo se reproduce en nuestra muestra (tanto en estudiantes como en académicos) y coincide con evidencia anterior (Baeza Reyes \& Lamadrid Álvarez, 2019). Arza et al. (2014) sostienen que las alumnas son un grupo con menor apoyo familiar y que pueden presentar menos satisfacción con las funciones del profesorado, lo que contribuye a indicar que el abordaje de los estudiantes, por parte de los docentes, debe incorporar diferencias de género.

En términos generales, las relaciones que se establecen con los estudiantes deben trascender la relación dentro del aula, sin descuidarla, por cierto, buscando formas de desarrollar relaciones más cercanas con los estudiantes y con ello lograr que reconozcan lo relevante de su formación. Aunque la disposición de los académicos puede ser negativa a la necesidad de estimular y motivar permanente a sus estudiantes a desarrollar el compromiso autónomo con su propia formación. Existe evidencia de que los estudiantes tienen una mejor relación con los profesores a los que demandan más apoyo (Arza, De Salvador \& Mascarenhas, 2014).

La importancia de promover este tipo de relaciones estudiante-docente va más allá del proceso de enseñanza de contenidos en sí y la búsqueda de la convivencia armónica. Promover la formación del profesorado en competencias relacionales que fomenten por ejemplo la tutoría proactiva - en contraposición a la respuesta actual reactiva - para mejorar la enseñanza, prevenir los fracasos y promover la mejora en el rendimiento académico, asociado a las desigualdades de partida que tiene el alumnado de las universidades públicas (Arza, De Salvador \& Mascarenhas, 2014; Salazar-Botello, Mendoza-Llanos \& Muñoz-Jara, 2020).

\section{Referencias}

Alarcón-Quinapanta, M. del R., Freire-Lescano, L. R., Pérez-Barral, O., Frías-Jiménez, R. A., \& Nogueira-Rivera, D. (2019). Medición del rendimiento del Talento Humano en Instituciones de Educación Superior: producción científica. Ingeniería Industrial, 40(1), 24-36.

Allard, R. (1998). Las nuevas tecnologías en el contexto de las políticas de fomento de calidad de la Educación Superior. Santiago: Consejo Superior de Educación de Chile.

Ángel-Macías, M. A., Ruiz-Díaz, P., \& Rojas-Soto, E. (2017). Propuesta de competencias profesionales para docentes de programas de salud en educación superior. Revista de La Facultad de Medicina, 65(4), 595-600. https://doi.org/10.15446/revfacmed.v65n4.58620

Arza, N., De Salvador, X. \& Mascarenhas, S. (2014). La función tutorial del profesorado: estudio evaluativo en tres universidades federales brasileñas. Revista Electrónica Interuniversitaria de Formación del Profesorado, 17(3), 105-.-121. DOI: http://dx.doi.org/10.6018/reifop.17.3.204081

Asún Dieste, S., Rapún López, M., \& Romero Martín, M. R. (2019). Percepciones de Estudiantes Universitarios sobre una Evaluación Formativa en el Trabajo en Equipo. Revista Iberoamericana de Evaluación Educativa, 12(1), 175-192. 
https://doi.org/10.15366/riee2019.12.1.010

Baeza Reyes, A \& Lamadrid Álvarez, S. (2019). ¿Igualdad en la academia? Barreras de género e iniciativas en una universidad pública (2013-2018). Pensamiento Educativo, 56 (1), 1-17, doi: 10.7764/PEL.56.1.2019.9

Blašková, M., Blaško, R., \& Kucharčíková, A. (2014). Competences and Competence Model of University Teachers. Procedia - Social and Behavioral Sciences, 159, 457-467. https://doi.org/10.1016/j.sbspro.2014.12.407

Bok, D. (1992). Educación Superior. Argentina: El Ateneo.

Bozu, Z., \& Canto, P. (2009). El profesorado universitario en la sociedad del conocimiento: competencias profesionales docentes . Revista de Formación e Innovación Educativa Universitaria, 2(2), 87-97. $\quad$ Retrieved from http://padula.detodoproducciones.com.ve/IS3_1_El profesorado universitario.pdf

Chadwick, C. (1983). Teorías del aprendizaje para el docente. Santiago, Chile: Universitaria.

Clemente-Ricolfe, J., \& Escribá-Pérez, C. (2013). Análisis de la percepción de las competencias genéricas adquiridas en la universidad. Revista de Educación, (362), 535-561. https://doi.org/10.4438/1988-592X-RE-2013-362-241

Corvalán, Ó. (2009). Calidad y evaluación de los aprendizajes y competencias en la Educación Superior. Revista Electrónica de Desarrollo de Competencias (REDEC), 1(3), 5-28. Retrieved from http://www.educandus.cl/ojs/index.php/fcompetencias/article/view/35/36

Díaz, J., \& Márquez, J. (2007). Estimación del potencial de competencias en la implementación de ejes transversales en instituciones de Educación Superior. Sapiens: Revista Universitaria de Investigación, 205-224.

Domínguez, S. (2012). Propuesta para el cálculo del Alfa Ordinal y Theta de Armor. Revista de Investigación en Psicología, 15(1), 213-217.

Etchegaray, P., Pascual, G., \& Calderón, O. (2018). Enseñanza de competencias genéricas en cursos de formación general de una universidad chilena: La visión de estudiantes y docentes. Revista Internacional de Investigación En Ciencias Sociales, 14(1), 77-88. https://doi.org/10.18004/riics.2018.julio.077-088

Erceg-Hurn, D. M., \& Mirosevich, V. M. (2008). Modern Robust Statistical Methods: An Easy Way to Maximize the Accuracy and Power of Your Research. American Psychologist, 63(7), 591-601. https://doi.org/10.1037/0003-066X.63.7.591

Fernandes, D. J., Sotolongo, M., \& Martínez, C. C. (2016). La evaluación del desempeño por competencias: Percepciones de docentes y estudiantes en la educación superior. Formacion Universitaria, 9(5), 15-24. https://doi.org/10.4067/S0718-50062016000500003

Fernández, G. (2007). Las Competencias: Clave para una Gestión Integrada de los Recursos Humanos. España: Deusto.

Fugellie, B. (2009). Educar el ser: La urgencia del cambio. Revista Electrónica de Desarrollo de $\begin{array}{lllll}\text { Competencias } \quad \text { (REDEC), } & \text { 29-34. } & \text { Retrieved from }\end{array}$ 
http://dta.utalca.cl/ojs/index.php/fcompetencias/article/view/36/39

Fugellie, B., Rodríguez, E., \& Yupanqui, A. (2009). Reflexiones sobre competencias y formación universitaria. Revista Electrónica de Desarrollo de Competencias (REDEC), 1(3), 56-63. Retrieved from http://www.educandus.cl/ojs/index.php/fcompetencias/article/view/38/41

Gomez, Ezequiel (2016). ¿Buenos profesores? La voz de los estudiantes de escuelas secundarias en contextos urbanos marginales. Pensamiento Educativo 53 (2), 1-12. doi: 10.7764/PEL.53.2.2016.7

González, J., \& Wagenaar, R. (2003). Tuning Educational Structures in Europe. Informe final. Fase 1. Universidad de Deusto y Universidad de Groningen.

González, R., \& González, V. (2007). Diagnóstico de necesidades y estrategias de formación docente en las Universidad. Revista Iberoamericana de Educación, 43(6).

Gutiérrez-Diez, M. del C., Piñón Howlet, L. C., \& Sapién Aguilar, A. L. (2020). Competencias docentes: brecha entre teoría y percepciones en la Universidad Autónoma de Chihuahua. RIDE Revista Iberoamericana Para La Investigación y El Desarrollo Educativo, 10(20). https://doi.org/10.23913/ride.v10i20.647

Gutiérrez, S., \& de Pablos, C. (2010). Análisis y evaluación de la gestión por competencias en el ámbito empresarial y su aplicación a la universidad. Revista Complutense de Educación, 21(2), 323-343. Retrieved from https://revistas.ucm.es/index.php/RCED/article/viewFile/RCED1010220323A/15112

Huerta, J., Pérez, I., \& Castellanos, A. (n.d.). Desarrollo curricular por competencias profesionales integrales.

Hurtado Espinoza, A. K. \& Madueño Serrano, M.L. (2017). Propiedades psicométricas del instrumento prácticas derivadas del aprendizaje social del profesorado. Revista Electrónica Interuniversitaria de Formación del Profesorado, 20 (3), 93---106. DOI: http://dx.doi.org/10.6018/reifop.20.3.291731

Ibáñez-Martín, J. A. (1990). Dimensiones de la Competencia Profesional del Profesor de Universidad. Revista Española de Pedagogía, 48(186), 239-280.

Irigoyen, J., Jiménez, M., \& Acuña, K. (2011). Competencias y Educación Superior. Revista Mexicana de Investigación Educativa (RMIE), 16(48), 243-266.

Jiménez, Y., Hernández, J., \& González, M. (2013). Competencias profesionales en la educación superior: justificación, evaluación y análisis. Innovación Educativa, 13(61), 45-65.

Le Boterf, G. (2000). Ingeniería de las Competencias. Barcelona: Gestión 2000.

Levy-Leboyer, C. (1995). Gestión de las Competencias. Barcelona: Gestión 2000.

López, A. B., González, I., \& De León, C. (2014). Perfil de un buen docente. Aplicación de un protocolo de evaluación de las competencias del profesorado universitario. Revista Electrónica Interuniversitaria de Formación Del Profesorado, 17(1), 133-148. https://doi.org/http://dx.doi.org/10.6018/reifop.17.1.190531 
Martínez, A. (2008). Competencias docentes del profesorado universitario de calidad. Primer Coloquio de Investigación Muldisciplinaria. Facultad de Estudios Profesionales, Acatlán, UNAM. México.

McCauley, C., Ohlott, P., \& Ruderman, M. (1989). On the job development: a conceptual model and prelimiary investigation. Jal of Managerial Issues, 1(2), 142-158.

McClelland, D. C. (1973). Testing for competence rather than for "intelligence." American Psychologist, 28(1), 1-14. Retrieved from http://www.ncbi.nlm.nih.gov/entrez/query.fcgi?cmd=Retrieve \&db=PubMed\&dopt=Citatio n\&list_uids=4684069

Mertens, L. (1996). Competencia Laboral: Sistemas, Surgimiento y Modelos. Montevideo: Cinterfor.

Moreno Murcia, J. A., \& Silveira Torregrosa, Y. (2015). Perfiles motivacionales de estudiantes universitarios. Procesos de estudio y satisfacción con la vida. Revista Electrónica Interuniversitaria de Formación Del Profesorado, 18(3), 169. https://doi.org/10.6018/reifop.18.3.200441

Oliveros, L. (2006). Identificación de competencias: una estrategia para la formación en el Espacio Europeo de Educación Superior. Revista Complutense de Educación, 17(1), 101$118 . \quad$ Retrieved from http://revistas.ucm.es/index.php/RCED/article/viewFile/RCED0606120101A/15882

Oyanedel, J. C., Vargas, S., Mella, C., \& Páez, D. (2017). Cálculo de confiabilidad a través del uso del coeficiente Omega de McDonald. Revista Médica de Chile, 145(2), 272-273. https://doi.org/https://doi.org/10.4067/S0034-98872017000200018

Pedraja, L., Rodríguez, E., \& Villarroel, C. (2012). Los estilos de liderazgo y la gestión de recursos humanos: una reflexión acerca del impacto sobre el desempeño de los estudiantes en la educación media. Ingeniare. Revista Chilena de Ingeniería, 20(3), 376-385.

Porto, A.M \& Mosteiro, M.J. (2014). Innovación y calidad en la formación del profesorado universitario. Revista Electrónica Interuniversitaria de Formación del Profesorado, 17(3), 141-156 DOI: http://dx.doi.org/10.6018/reifop.17.3.204101

Posada, R. (2004). Formación superior basada en competencias, interdisciplinariedad y trabajo autónomo del estudiante. Revista Iberoamericana de Educación, 1-33.

Quinn, R., Faerman, S., Thompson, M., \& McGrath, M. (1994). Maestría en la Gestión de Organizaciones: Un modelo operativo de Competencias. (1st ed.). Díaz de Santos.

Riveros Munévar, F., Bohórquez Borda, D., López Castillo, S., \& Sepúlveda Rodríguez, E. (2015). Diseño y validación de un instrumento para medir las actitudes frente a la labor profesional del psicólogo. Revista Iberoamericana de Psicología: Ciencia y Tecnología, $8(2), 55-65$.

Rumbo, M. B. (1998). La calidad de la enseñanza universitaria y el desarrollo profesional de su profesorado. A Coruña: Grupo Editorial Universitario.

Sachau D. Resurrecting the Motivation-Hygiene Theory: Herzberg and the positive psychology 
movement. Human Resource Development Review. 2007; 6(4):377-93.

Salas, W. (2005). Formación por Competencias en Educación Superior. Una aproximación conceptual a propósito del caso colombiano. Revista Iberoamericana de Educación, 36(9).

Salazar, C., \& Chiang, M. (2007). Competencias y desempeño laboral. Taller de Empleo Regional, 30, 21-37. Retrieved from http://www.tallerdeempleo.cl/informes/ter030.pdf

Salazar, C., Chiang, M., \& Muñoz, Y. (2016). Competencias docentes en la educación superior: Un estudio empírico en la Universidad del Bío-Bío. Revista Actualidades Investigativas En Educación, 6(1), 1-28. https://doi.org/http://dx.doi.org/10.15517/aie.v16i1.22383

Salazar, M., Mendoza-Llanos, R., \& Muñoz, Y. (2020). Impacto diferenciado del tiempo de formación universitaria según institución de educación media en el desarrollo de habilidades sociales. Propósitos y Representaciones, 8(2). Doi: http://dx.doi.org/10.20511/pyr2020.v8n2.416

Salazar, C., \& Yobánolo, R. (2002). Identificación de competencias del docente de excelencia de la Universidad del Bío-Bío. Theoria, 11(1), 77-85. Retrieved from http://redalyc.uaemex.mx/redalyc/src/inicio/ArtPdfRed.jsp?iCve=29901111

Segura, M. (2004). Hacia un perfil del docente Universitario. Ciencias de La Educación, 1(23), 9-28. Retrieved from http://servicio.cid.uc.edu.ve/educacion/revista/a4n23/23-1.pdf

Spencer, L. M., \& Spencer, S. M. (1993). Competence at work: Models for superior performance. Retrieved from http://www.books24x7.com/marc.asp?isbn=047154809X

Tigelaar, D., Dolmans, D., Wolfhagen, I., \& Van Der Vleuten, C. (2004). The development and validation of a framework for teaching competencies in higher education. Higher Education, $48(2), 253-268$.

Vargas, J. G. (2017). Las reglas cambiantes de la competitividad global en el nuevo milenio. Red Internacional de Investigadores En Competitividad Memoria Del I Congreso, 4(1), 21002119. Retrieved from http://riico.net/index.php/riico/issue/view/14

Villarroel, V. A., \& Bruna, D. V. (2017). Competencias Pedagógicas que Caracterizan a un Docente Universitario de Excelencia: Un Estudio de Caso que Incorpora la Perspectiva de Docentes y Estudiantes. Formación Universitaria, 10(4), 75-96. https://doi.org/10.4067/S0718-50062017000400008

Yáñez, Ó. J., Sánchez, C. O., \& Held, B. H. (2016). Atributos de una docencia de calidad en la educación superior: Una revisión sistemática. Estudios Pedagógicos, 42(3), 483-506. https://doi.org/10.4067/s0718-07052016000400026

Zabalza, M. (2003). Competencias Docentes del Profesorado Universitario: Calidad y Desarrollo Profesional. Madrid: Ediciones Nancea. 